\title{
Existence of stable standing waves for the Schrödinger-Choquard equation
}

Kun Liu' ${ }^{1 *}$ and Cunqin Shi

${ }^{\text {*Correspondence: }}$ liukunws@163.com

'School of Mathematics and Statistic, Longdong University, Qingyang, P.R. China

\section{照 Springer}

\begin{abstract}
In this paper, by variational methods and the profile decomposition of bounded sequences in $H^{1}$ we study the existence of stable standing waves for the Schrödinger-Choquard equation with an $L^{2}$-critical nonlinearity. Our results extend some earlier results.
\end{abstract}

Keywords: Schrödinger-Choquard equation; $L^{2}$-critical nonlinearity; Orbital stability of standing waves

\section{Introduction}

In this paper, we consider the existence of stable standing waves for the following nonlinear Schrödinger-Choquard equation:

$$
\left\{\begin{array}{l}
i \psi_{t}+\Delta \psi=\lambda_{1}|\psi|^{p_{1}} \psi+\lambda_{2}\left(I_{\alpha} *|\psi|^{p_{2}}\right)|\psi|^{p_{2}-2} \psi, \quad(t, x) \in[0, T) \times \mathbb{R}^{N} \\
\psi(0, x)=\psi_{0}(x)
\end{array}\right.
$$

where $\psi(t, x):[0, T) \times \mathbb{R}^{N} \rightarrow \mathbb{C}$ is a complex-valued function, $0<T \leq \infty, N \geq 3, \psi_{0} \in H^{1}$, $0<p_{1}<\frac{4}{N}, 1+\frac{\alpha}{N}<p_{2}<1+\frac{2+\alpha}{N-2}, \lambda_{1}, \lambda_{2} \in \mathbb{R}, I_{\alpha}: \mathbb{R}^{N} \rightarrow \mathbb{R}$ is the Riesz potential defined by

$$
I_{\alpha}(x)=\frac{\Gamma\left(\frac{N-\alpha}{2}\right)}{\Gamma\left(\frac{\alpha}{2}\right) \pi^{N / 2} 2^{\alpha}|x|^{N-\alpha}}
$$

with $\max \{0, N-4\}<\alpha<N$, and $\Gamma$ is the gamma function.

One of the motivations for studying this problem is that this equation is not scale invariant. When $\lambda_{1}=0$ and $p_{2}>0$, for the nonlinear Choquard equation

$$
i \psi_{t}+\Delta \psi=\lambda_{2}\left(I_{\alpha} *|\psi|^{p_{2}}\right)|\psi|^{p_{2}-2} \psi
$$

there is a scaling transform that leaves it invariant. More precisely, the map

$$
\psi(t, x) \mapsto \lambda^{-\frac{\alpha+2}{2 p_{2}-2}} \psi\left(\frac{t}{\lambda^{2}}, \frac{x}{\lambda}\right)
$$

maps a solution of (1.2) to another solution of (1.2). When $p_{2}=1+\frac{2+\alpha}{N}$, the scaling (1.3) leaves the mass invariant. Therefore, the nonlinearity $\left(I_{\alpha} *|\psi|^{p_{2}}\right)|\psi|^{p_{2}-2} \psi$ is called $L^{2}$ critical.

(c) The Author(s) 2018. This article is distributed under the terms of the Creative Commons Attribution 4.0 International License (http://creativecommons.org/licenses/by/4.0/), which permits unrestricted use, distribution, and reproduction in any medium, provided you give appropriate credit to the original author(s) and the source, provide a link to the Creative Commons license, and indicate if changes were made. 
When $p_{2}=2$, equation (1.2) simplifies to the well-known Hartree equation. The Cauchy problem of (1.1) has been extensively investigated in [1-12]. The local well-posedness and asymptotical behavior of the solutions for (1.1) have been studied in [1,5]. Chen and Guo [2] studied the instability of standing waves. Miao et al. [10] studied the dynamical properties of the blowup solutions with minimal mass in the $L^{2}$-critical case. The soliton dynamics has been investigated in [3].

When $0<\alpha<N$ and $1+\frac{\alpha}{N}<p_{2}<\frac{N+\alpha}{N-2}$, under the assumption that the local wellposedness holds for (1.2), Chen and Guo [2] derived the existence of blowup solutions and the instability of standing waves. When $0<\alpha<N$ and $1+\frac{\alpha}{N}<p_{2}<1+\frac{2+\alpha}{N}$, the soliton dynamics of (1.1) has been investigated in [13] if the solution $\psi$ of (1.1) is in $C\left([0, \infty), H^{2}\right) \cap C^{1}\left((0, \infty), L^{2}\right)$. Feng and Yuan [14] systematically studied the Cauchy problem (1.2) for general $\max \{0, N-4\}<\alpha<N$ and $2 \leq p_{2}<\frac{N+\alpha}{N-2}$. More precisely, they study the local and global well-posedness, finite-time blowup, and the dynamics of blowup solutions. The sharp threshold of global existence and instability of standing wave for (1.2) with a harmonic potential have been investigated in [15].

Recently, in the $L^{2}$-subcritical case, that is, where $1+\frac{\alpha}{N}<p_{2}<1+\frac{2+\alpha}{N}$, Wang et al. [16] studied the orbital stability of standing waves for (1.2) with $\lambda_{1}=0$ and $\lambda_{2}=-1$. However, in this paper, the study of the stability of standing waves relies heavily on the scale invariance for (1.2). Thus, adding an $L^{2}$-subcritical perturbation to (1.2), which destroys the scale invariance, is of particular interest. In addition, the study of orbitally stable standing waves for nonlinear Schrödinger equations is in the $L^{2}$-subcritical case; see [16-21]. In the $L^{2}$-critical case, the solution of (1.1) may blow up; see [1, 14, 22, 23]. It yields that the standing waves may be unstable. This brings some essential difficulties to the study of stable standing waves for (1.1) in the $L^{2}$-critical case.

To study the stability of standing waves of (1.1), we first make the following assumption for the local well-posedness for (1.1).

Assumption 1 Let $\psi_{0} \in H^{1}, N \geq 3,0<p_{1}<\frac{4}{N}$, and $1+\frac{\alpha}{N}<p_{2}<1+\frac{2+\alpha}{N-2}$. Then there exists $T=T\left(\left\|\psi_{0}\right\|_{H^{1}}\right)$ such that $(1.1)$ admits a unique solution $\psi \in C\left([0, T], H^{1}\right)$. Let $\left[0, T^{*}\right)$ be the maximal interval on which the solution $\psi(t)$ is well-defined: if $T^{*}<\infty$, then $\|\psi(t)\|_{H^{1}} \rightarrow$ $\infty$ as $t \uparrow T^{*}$. Moreover, for all $0 \leq t<T^{*}$, the solution $\psi(t)$ satisfies the conservation of mass and energy:

$$
\begin{aligned}
& \|\psi(t)\|_{L^{2}}=\left\|\psi_{0}\right\|_{L^{2}}, \\
& E(\psi(t))=E\left(\psi_{0}\right),
\end{aligned}
$$

where $E(\psi(t))$ is defined by

$$
\begin{aligned}
E(\psi(t)):= & \frac{1}{2} \int_{\mathbb{R}^{N}}|\nabla \psi(t, x)|^{2} d x+\frac{\lambda_{1}}{p_{1}+2} \int_{\mathbb{R}^{N}}|\psi(t, x)|^{p_{1}+2} d x \\
& +\frac{\lambda_{2}}{2 p_{2}} \int_{\mathbb{R}^{N}}\left(I_{\alpha} *|\psi|^{p_{2}}\right)(t, x)|\psi(t, x)|^{p_{2}} d x .
\end{aligned}
$$

Remark When $0<p_{1}<\frac{4}{N}$ and $2 \leq p_{2}<1+\frac{2+\alpha}{N-2}$, this assumption can be easily proved by Strichartz's estimates and a fixed point argument $[1,14]$. When $1+\frac{\alpha}{N}<p_{2}<2$, we deduce from inequality (2.1) that $\int_{\mathbb{R}^{N}}\left(I_{\alpha} *|\psi|^{p_{2}}\right)|\psi|^{p_{2}} d x$ is well-defined for $\psi \in H^{1}$. Thus, we 
assume that the local well-posedness of (1.1) holds for $\frac{N+\alpha}{N}<p_{2}<2$. However, we cannot prove this result because the nonlinearity $\left(I_{\alpha} *|\psi|^{p_{2}}\right)|\psi|^{p_{2}-2} \psi$ is singular when $\frac{N+\alpha}{N}<p<2$. Consequently, the case of $\frac{N+\alpha}{N}<p_{2}<2$ will be the object of a future investigation.

Under this assumption, by using variational methods and the profile decomposition of bounded sequences in $H^{1}$ we can obtain the following theorem.

Theorem 1.1 Let $N \geq 3, \lambda_{1}=-1, \lambda_{2}=-1,0<p_{1}<\frac{4}{N}, p_{2}=1+\frac{2+\alpha}{N}$, and $\left\|\psi_{0}\right\|_{L^{2}}<\|Q\|_{L^{2}}$, where $Q$ is a ground state of

$$
-\Delta Q+Q=\left(I_{\alpha} *|Q|^{p_{2}}\right)|Q|^{p_{2}-2} Q \text { in } \mathbb{R}^{N}
$$

If Assumption 1 holds, then the standing waves of (1.1) are orbitally stable.

This paper is organized as follows: in Sect. 2, we first collect some lemmas such as the Hardy-Littlewood-Sobolev inequality and the profile decomposition of bounded sequences in $H^{1}$. In Sect. 3, we study the orbital stability of standing waves of (1.1).

\section{Preliminaries}

In this section, we will recall some preliminary results. We first recall the HardyLittlewood-Sobolev inequality.

Lemma 2.1 Let $0<\lambda<N$ and $s, r>1$ be constants such that

$$
\frac{1}{r}+\frac{1}{s}+\frac{\lambda}{N}=2
$$

Assume that $f \in L^{r}$ and $g \in L^{s}$. Then

$$
\left|\int_{\mathbb{R}^{N}} \int_{\mathbb{R}^{N}} f(x)\right| x-\left.y\right|^{-\lambda} g(y) d x d y \mid \leq C(N, s, \lambda)\|f\|_{L^{r}}\|g\|_{L^{s}}
$$

See Lieb [24] for the proof.

Next, we recall a useful result, which gives the best constant in a Gagliardo-Nirenbergtype inequality; see [14].

Lemma 2.2 The best constant in the Gagliardo-Nirenberg-type inequality

$$
\int_{\mathbb{R}^{N}}\left(I_{\alpha} *|u|^{p}\right)|u|^{p} d x \leq C_{\alpha, p}\left(\int_{\mathbb{R}^{N}}|\nabla u|^{2} d x\right)^{\frac{N p-N-\alpha}{2}}\left(\int_{\mathbb{R}^{N}}|u|^{2} d x\right)^{\frac{N+\alpha-N p+2 p}{2}}
$$

is

$$
C_{\alpha, p}=\frac{2 p}{2 p-N p+N+\alpha}\left(\frac{2 p-N p+N+\alpha}{N p-N-\alpha}\right)^{\frac{N p-N-\alpha}{2}}\|Q\|_{L^{2}}^{2-2 p},
$$

where $Q$ is the ground state solution of (1.5). In particular, in the $L^{2}$-critical case $p=1+\frac{2+\alpha}{N}$, $C_{\alpha, p}=p\|Q\|_{L^{2}}^{2-2 p}$. 
We further summarize some results about the ground state of (1.5), which is very important in the study of the dynamics of blowup solutions to (1.1).

Lemma $2.3([13,25])$ Let $\alpha \in(0, N)$ and $1+\frac{\alpha}{N}<p<1+\frac{2+\alpha}{N-2}$. Then (1.5) admits a ground state solution $Q$ in $H^{1}$. Every ground state $Q$ of $(1.5)$ is in $L^{1} \cap C^{\infty}$, it has fixed sign, and there exist $x_{0} \in \mathbb{R}^{N}$ and a monotone real function $v \in C^{\infty}(0, \infty)$ such that, for every $x \in \mathbb{R}^{N}$, $Q(x)=v\left(\left|x-x_{0}\right|\right)$. Moreover, the $L^{2}$-norm of any ground state $Q$ of $(1.5)$ is the same.

Next, we recall the profile decomposition of bounded sequences in $H^{1}$ proposed by Hmidi and Keraani [26], which is important in studying the stability of standing waves to $(1.1)$.

Lemma 2.4 Let $\left\{u_{n}\right\}_{n=1}^{\infty}$ be a bounded sequence in $H^{1}$. Then there exist a subsequence of $\left\{u_{n}\right\}_{n=1}^{\infty}$ (still denoted by $\left\{u_{n}\right\}_{n=1}^{\infty}$ ), a family $\left\{x_{n}^{j}\right\}_{j=1}^{\infty}$ of sequences in $\mathbb{R}^{N}$, and a sequence $\left\{U^{j}\right\}_{j=1}^{\infty}$ in $H^{1}$ such that

(i) for every $k \neq j,\left|x_{n}^{k}-x_{n}^{j}\right| \rightarrow+\infty$ as $n \rightarrow \infty$;

(ii) for every $l \geq 1$ and every $x \in \mathbb{R}^{N}$, we have

$$
u_{n}(x)=\sum_{j=1}^{l} U^{j}\left(x-x_{n}^{j}\right)+r_{n}^{l}
$$

with $\lim \sup _{n \rightarrow \infty}\left\|r_{n}^{l}\right\|_{L^{q}} \rightarrow 0$ as $l \rightarrow \infty$ for every $q \in\left(2, \frac{2 N}{N-2}\right)$. Moreover,

$$
\begin{aligned}
& \left\|u_{n}\right\|_{L^{2}}^{2}=\sum_{j=1}^{l}\left\|U^{j}\right\|_{L^{2}}^{2}+\left\|r_{n}^{l}\right\|_{L^{2}}^{2}+o(1), \\
& \left\|\nabla u_{n}\right\|_{L^{2}}^{2}=\sum_{j=1}^{l}\left\|\nabla U^{j}\right\|_{L^{2}}^{2}+\left\|\nabla r_{n}^{l}\right\|_{L^{2}}^{2}+o(1), \\
& \left.\int_{\mathbb{R}^{N}} I_{\alpha} *\left|\sum_{j=1}^{l} U^{j}\left(\cdot-x_{n}^{j}\right)\right|\right|^{p}\left|\sum_{j=1}^{l} U^{j}\left(x-x_{n}^{j}\right)\right|^{p} d x \\
& =\sum_{j=1}^{l} \int_{\mathbb{R}^{N}} I_{\alpha} *\left|U^{j}\left(\cdot-x_{n}^{j}\right)\right|^{p}\left|U^{j}\left(x-x_{n}^{j}\right)\right|^{p} d x+o(1),
\end{aligned}
$$

where $\circ(1)=o_{n}(1) \rightarrow 0$ as $n \rightarrow \infty$.

Remark (2.6) has been proved in [14].

Finally, we have the following global existence of (1.1).

Theorem 2.5 Let $\psi_{0} \in H^{1}, \lambda_{1}=-1, \lambda_{2}=-1,0<p_{1}<\frac{4}{N}$, and $p_{2}=1+\frac{2+\alpha}{N}$. Assume that $Q$ is the ground state solution of (1.5) and $\left\|\psi_{0}\right\|_{L^{2}}<\|Q\|_{L^{2}}$. If Assumption 1 holds, then the solution $\psi(t)$ of (1.1) exists globally.

Proof Recall the Gagliardo-Nirenberg inequality

$$
\int_{\mathbb{R}^{N}}|u(x)|^{p_{1}+2} d x \leq C\|u\|_{L^{2}}^{p_{1}+2-\frac{N p_{1}}{2}}\|\nabla u\|_{L^{2}}^{\frac{N p_{1}}{2}} \quad \text { for } u \in H^{1} .
$$


Hence we deduce from (1.4) and (2.2) that

$$
\begin{aligned}
E\left(\psi_{0}\right)= & E(\psi(t)) \\
= & \frac{1}{2} \int_{\mathbb{R}^{N}}|\nabla \psi(t, x)|^{2} d x-\frac{1}{p_{1}+2} \int_{\mathbb{R}^{N}}|\psi(t, x)|^{p_{1}+2} d x \\
& -\frac{1}{2 p_{2}} \int_{\mathbb{R}^{N}}\left(I_{\alpha} *|\psi|^{p_{2}}\right)(t, x)|\psi(t, x)|^{p_{2}} d x \\
\geq & \left(\frac{1}{2}-\frac{\left\|\psi_{0}\right\|_{L^{2}}^{2 p_{2}-2}}{2\|Q\|_{L^{2}}^{2 p_{2}-2}}\right)\|\nabla \psi(t)\|_{L^{2}}^{2}-C\left\|\psi_{0}\right\|_{L^{2}}^{p_{1}+2-\frac{N p_{1}}{2}}\|\nabla \psi(t)\|_{L^{2}}^{\frac{N p_{1}}{2}} .
\end{aligned}
$$

Since $0<p_{1}<\frac{4}{N}$, it follows that $\frac{N p_{1}}{2}<2$. Thus, we infer from Young's inequality that, for all $0<\varepsilon<\frac{1}{2}$, there exists a constant $C(\varepsilon, M)$ such that

$$
C\left\|\psi_{0}\right\|_{L^{2}}^{p_{1}+2-\frac{N p_{1}}{2}}\|\nabla \psi(t)\|_{L^{2}}^{\frac{N p_{1}}{2}} \leq \varepsilon\|\nabla \psi(t)\|_{L^{2}}^{2}+C\left(\varepsilon,\left\|\psi_{0}\right\|_{L^{2}}\right)
$$

This implies that

$$
E\left(\psi_{0}\right) \geq\left(\frac{1}{2}-\frac{\left\|\psi_{0}\right\|_{L^{2}}^{2 p_{2}-2}}{2\|Q\|_{L^{2}}^{2 p_{2}-2}}-\varepsilon\right)\|\nabla \psi(t)\|_{L^{2}}^{2}-C\left(\varepsilon,\left\|\psi_{0}\right\|_{L^{2}}\right) .
$$

This, together with $\left\|\psi_{0}\right\|_{L^{2}}<\|Q\|_{L^{2}}$, implies that there exists a constant $C$ such that $\|\nabla \psi(t)\|_{L^{2}} \leq C$ for all $t>0$. Therefore, the solution $\psi(t)$ of (1.1) exists globally.

\section{Orbital stability of standing waves}

First, let $N \geq 3, \lambda_{1}=-1, \lambda_{2}=-1,0<p_{1}<\frac{4}{N}, p_{2}=1+\frac{2+\alpha}{N}$, and $0<M<\|Q\|_{L^{2}}^{2}$, where $Q$ is the ground state solution of (1.5). We can define the variational problem

$$
d_{M}:=\inf _{\left\{u \in H^{1} ;\|u\|_{L^{2}}^{2}=M\right\}} E(u)
$$

where $E(u)$ is the energy functional defined in (1.4). In the following theorem, we apply the profile decomposition of bounded sequences in $H^{1}$ to solve the variational problem (3.1).

Theorem 3.1 Let $N \geq 3, \lambda_{1}=-1, \lambda_{2}=-1,0<p_{1}<\frac{4}{N}, p_{2}=1+\frac{2+\alpha}{N}$, and $0<M<\|Q\|_{L^{2}}^{2}$, where $Q$ is the ground state solution of (1.5). Then there exists $u_{0} \in H^{1}$ such that $d_{M}=$ $E\left(u_{0}\right)$.

Proof First, we show that the variational problem (3.1) is well-defined and there exists $C_{0}>0$ such that

$$
d_{M} \leq-C_{0}<0
$$


Indeed, we deduce from (1.4), (2.2), and (2.7) that there exists a constant $C$ such that

$$
\begin{aligned}
E(u):= & \frac{1}{2} \int_{\mathbb{R}^{N}}|\nabla u(x)|^{2} d x-\frac{1}{p_{1}+2} \int_{\mathbb{R}^{N}}|u(x)|^{p_{1}+2} d x \\
& -\frac{1}{2 p_{2}} \int_{\mathbb{R}^{N}}\left(I_{\alpha} *|u|^{p_{2}}\right)(x)|u(x)|^{p_{2}} d x \\
\geq & \left(\frac{1}{2}-\frac{\|u\|_{L^{2}}^{2 p_{2}-2}}{2\|Q\|_{L^{2}}^{2 p_{2}-2}}\right)\|\nabla u\|_{L^{2}}^{2}-C\|u\|_{L^{2}}^{p_{1}+2-\frac{N p_{1}}{2}}\|\nabla u\|_{L^{2}}^{\frac{N p_{1}}{2}} .
\end{aligned}
$$

Since $\frac{N p_{1}}{2}<2$, it follows from Young's inequality that, for all $0<\varepsilon<\frac{1}{2}$, there exists a constant $C(\varepsilon, M)$ such that

$$
C\|u\|_{L^{2}}^{p_{1}+2-\frac{N p_{1}}{2}}\|\nabla u\|_{L^{2}}^{\frac{N p_{1}}{2}} \leq \varepsilon\|\nabla u\|_{L^{2}}^{2}+C(\varepsilon, M) .
$$

This implies that

$$
E(u) \geq\left(\frac{1}{2}\left(1-\frac{\|u\|_{L^{2}}^{2 p_{2}-2}}{\|Q\|_{L^{2}}^{2 p_{2}-2}}\right)-\varepsilon\right)\|\nabla u\|_{L^{2}}^{2}-C(\varepsilon, M) .
$$

Therefore we deduce from the hypothesis $\|u\|_{L^{2}}^{2}=M<\|Q\|_{L^{2}}^{2}$ that $E(u)$ has a lower bound and the variational problem (3.1) is well-defined.

Now, let $u \in H^{s}$ be a fixed function, and let $\mu>0$. Set $u_{\mu}=\mu^{\frac{N}{2}} u(\mu x)$. It follows easily that

$$
\left\|u_{\mu}\right\|_{L^{2}}^{2}=\|u\|_{L^{2}}^{2}=M
$$

and

$$
\begin{aligned}
E\left(u_{\mu}\right)= & \frac{\mu^{2}}{2} \int_{\mathbb{R}^{N}}|\nabla u(x)|^{2} d x-\frac{\mu^{\frac{N p_{1}}{2}}}{p_{1}+2} \int_{\mathbb{R}^{N}}|u(x)|^{p_{1}+2} d x \\
& -\frac{\mu^{2}}{2 p_{2}} \int_{\mathbb{R}^{N}}\left(I_{\alpha} *|u|^{p_{2}}\right)(x)|u(x)|^{p_{2}} d x \\
= & \mu^{2}\left(\frac{1}{2} \int_{\mathbb{R}^{N}}|\nabla u(x)|^{2} d x-\frac{1}{2 p_{2}} \int_{\mathbb{R}^{N}}\left(I_{\alpha} *|u|^{p_{2}}\right)(x)|u(x)|^{p_{2}} d x\right) \\
& -\frac{\mu^{\frac{N p_{1}}{2}}}{p_{1}+2} \int_{\mathbb{R}^{N}}|u(x)|^{p_{1}+2} d x .
\end{aligned}
$$

On the other hand, by the sharp Gagliardo-Nirenberg inequality (2.2) and $\|u\|_{L^{2}}^{2}=M<$ $\|Q\|_{L^{2}}^{2}$ it follows that there exists $C_{1}>0$ such that

$$
\frac{1}{2} \int_{\mathbb{R}^{N}}|\nabla u(x)|^{2} d x-\frac{1}{2 p_{2}} \int_{\mathbb{R}^{N}}\left(I_{\alpha} *|u|^{p_{2}}\right)(x)|u(x)|^{p_{2}} d x \geq C_{1}>0 .
$$

Since $\frac{N p_{1}}{2}<2$, we can choose $\mu>0$ sufficiently small such that there exists $C_{0}>0$ such that $E\left(u_{\mu}\right) \leq-C_{0}<0$. Hence (3.2) is true. 
Second, let $\left\{u_{n}\right\}_{n=1}^{\infty}$ be a minimizing sequence of the variational problem (3.1) such that

$$
E\left(u_{n}\right) \rightarrow d_{M} \quad \text { and } \quad\left\|u_{n}\right\|_{L^{2}}^{2}=M
$$

This implies that, for $n$ large enough, $E\left(u_{n}\right)<d_{M}+1$. Thus, for all $0<\varepsilon<\frac{1}{2}\left(1-\frac{\|u\|_{L^{2}}^{2 p_{2}-2}}{\|Q\|_{L^{2}}^{2 p_{2}-2}}\right)$, we have

$$
\left(\frac{1}{2}\left(1-\frac{\|u\|_{L^{2}}^{2 p_{2}-2}}{\|Q\|_{L^{2}}^{2 p_{2}-2}}\right)-\varepsilon\right)\|\nabla u\|_{L^{2}}^{2} \leq d_{M}+1+C\left(\varepsilon,\|Q\|_{L^{2}}, M\right) .
$$

This yields that $\left\{u_{n}\right\}_{n=1}^{\infty}$ is bounded in $H^{1}$.

Third, applying the profile decomposition of bounded sequences in $H^{1}$, we will show that the infimum of the variational problem (3.1) can be attained. Apply Lemma 2.4 to the minimizing sequence $\left\{u_{n}\right\}_{n=1}^{\infty}$, which, up to a subsequence, can be decomposed as

$$
u_{n}(x)=\sum_{j=1}^{l} U^{j}\left(x-x_{n}^{j}\right)+r_{n}^{l}
$$

with $\lim \sup _{n \rightarrow \infty}\left\|r_{n}^{l}\right\|_{L q} \rightarrow 0$ as $l \rightarrow \infty$ for every $q \in\left(2, \frac{2 N}{N-2}\right)$.

Now, injecting (3.5) into the energy functional $E\left(u_{n}\right)$, it follows from (2.4)-(2.6) that

$$
E\left(u_{n}\right)=\sum_{j=1}^{l} E\left(U^{j}\right)+E\left(r_{n}^{l}\right)+o(1)
$$

as $n \rightarrow \infty$ and $l \rightarrow \infty$. For every $U^{j}(1 \leq j \leq l)$, take the scaling transform $U_{\mu_{j}}^{j}=\mu_{j} U^{j}$ with $\mu_{j}=\frac{\sqrt{M}}{\left\|U^{\prime}\right\|_{L^{2}}}$. It follows easily that

$$
\left\|U_{\mu_{j}}^{j}\right\|_{L^{2}}^{2}=M
$$

and

$$
\begin{aligned}
E\left(U_{\mu_{j}}^{j}\right)= & \frac{\mu_{j}^{2}}{2} \int_{\mathbb{R}^{N}}\left|\nabla U^{j}(x)\right|^{2} d x-\frac{\mu_{j}^{p_{1}+2}}{p_{1}+2} \int_{\mathbb{R}^{N}}\left|U^{j}(x)\right|^{p_{1}+2} d x \\
& -\frac{\mu_{j}^{2 p_{2}}}{2 p_{2}} \int_{\mathbb{R}^{N}}\left(I_{\alpha} *\left|U^{j}\right|^{p_{2}}\right)(x)\left|U^{j}(x)\right|^{p_{2}} d x \\
= & \mu_{j}^{2} E\left(U^{j}\right)-\frac{\mu_{j}^{2}\left(\mu_{j}^{p_{1}}-1\right)}{p_{1}+2} \int_{\mathbb{R}^{N}}\left|U^{j}(x)\right|^{p_{1}+2} d x \\
& -\frac{\mu_{j}^{2}\left(\mu_{j}^{2 p_{2}-2}-1\right)}{2 p_{2}} \int_{\mathbb{R}^{N}}\left(I_{\alpha} *\left|U^{j}\right|^{p_{2}}\right)(x)\left|U^{j}(x)\right|^{p_{2}} d x .
\end{aligned}
$$


This yields

$$
\begin{aligned}
E\left(U^{j}\right)= & \frac{E\left(U_{\mu_{j}}^{j}\right)}{\mu_{j}^{2}}+\frac{\left(\mu_{j}^{p_{1}}-1\right)}{p_{1}+2} \int_{\mathbb{R}^{N}}\left|U^{j}(x)\right|^{p_{1}+2} d x \\
& +\frac{\mu_{j}^{2 p_{2}-2}-1}{2 p_{2}} \int_{\mathbb{R}^{N}}\left(I_{\alpha} *\left|U^{j}\right|^{p_{2}}\right)(x)\left|U^{j}(x)\right|^{p_{2}} d x .
\end{aligned}
$$

Similarly, for the term $E\left(r_{n}^{l}\right)$, we obtain

$$
\begin{aligned}
E\left(r_{n}^{l}\right)= & \frac{\left\|r_{n}^{l}\right\|_{L^{2}}^{2}}{M} E\left(\frac{\sqrt{M}}{\left\|r_{n}^{l}\right\|_{L^{2}}} r_{n}^{l}\right)+\frac{\left(\left(\frac{\sqrt{M}}{\left\|r_{n}^{l}\right\|_{L^{2}}}\right)^{p_{1}}-1\right)}{p_{1}+2} \int_{\mathbb{R}^{N}}\left|r_{n}^{l}(x)\right|^{p_{1}+2} d x \\
& +\frac{\left(\frac{\sqrt{M}}{\left\|r_{n}^{l}\right\|_{L^{2}}}\right)^{2 p_{2}-2}-1}{2 p_{2}} \int_{\mathbb{R}^{N}}\left(I_{\alpha} *\left|r_{n}^{l}\right|^{p_{2}}\right)(x)\left|r_{n}^{l}(x)\right|^{p_{2}} d x+\circ(1) \\
\geq & \frac{\left\|r_{n}^{l}\right\|_{L^{2}}^{2}}{M} E\left(\frac{\sqrt{M}}{\left\|r_{n}^{l}\right\|_{L^{2}}} r_{n}^{l}\right)+\circ(1) .
\end{aligned}
$$

Since $\left\|U_{\mu_{j}}^{j}\right\|_{L^{2}}^{2}=\left\|\frac{\sqrt{M}}{\left\|r_{n}^{l}\right\|_{L^{2}}} r_{n}^{l}\right\|_{L^{2}}^{2}=M$, we deduce from the definition of $d_{M}$ that

$$
E\left(U_{\mu_{j}}^{j}\right) \geq d_{M} \quad \text { and } \quad E\left(\frac{\sqrt{M}}{\left\|r_{n}^{l}\right\|_{L^{2}}} r_{n}^{l}\right) \geq d_{M} .
$$

Thus we infer from (3.6), (3.8), and (3.9) that

$$
\begin{aligned}
E\left(u_{n}\right) \geq & \sum_{j=1}^{l}\left(\frac{E\left(U_{\mu_{j}}^{j}\right)}{\mu_{j}^{2}}+\frac{\left(\mu_{j}^{p_{1}}-1\right)}{p_{1}+2} \int_{\mathbb{R}^{N}}\left|U^{j}(x)\right|^{p_{+} 2} d x\right. \\
& \left.+\frac{\mu_{j}^{2 p_{2}-2}-1}{2 p_{2}} \int_{\mathbb{R}^{N}}\left(I_{\alpha} *\left|U^{j}\right|^{p_{2}}\right)(x)\left|U^{j}(x)\right|^{p_{2}} d x\right) \\
& +\frac{\left\|r_{n}^{l}\right\|_{L^{2}}^{2} E\left(\frac{\sqrt{M}}{M} r_{n}^{l}\right)+\circ(1)}{\left\|r_{n}^{l}\right\|_{L^{2}}} \\
\geq & \sum_{j=1}^{l} \frac{\left\|U^{j}\right\|_{L^{2}}^{2}}{M} d_{M}+\inf _{j \geq 1} \frac{\left(\mu_{j}^{p_{1}}-1\right)}{p_{1}+2}\left(\sum_{j=1}^{l} \int_{\mathbb{R}^{N}}\left|U^{j}(x)\right|^{p_{1}+2} d x\right) \\
& +\inf _{j \geq 1} \frac{\mu_{j}^{2 p_{2}-2}-1}{2 p_{2}}\left(\sum_{j=1}^{l} \int_{\mathbb{R}^{N}}\left(I_{\alpha} *\left|U^{j}\right|^{p_{2}}\right)(x)\left|U^{j}(x)\right|^{p_{2}} d x\right)+\frac{\left\|r_{n}^{l}\right\|_{L^{2}}^{2}}{M} d_{M}+\circ(1) \\
\geq & \sum_{j=1}^{l} \frac{\left\|U^{j}\right\|_{L^{2}}^{2}}{M} d_{M}+\frac{\left\|r_{n}^{l}\right\|_{L^{2}}^{2}}{M} d_{M} \\
& +\inf _{j \geq 1}\left(\mu_{j}^{a_{0}}-1\right)\left(\frac{1}{2 p_{2}} \int_{\mathbb{R}^{N}}\left(I_{\alpha} *\left|u_{n}\right|^{p_{2}}\right)(x)\left|u_{n}(x)\right|^{p_{2}} d x\right. \\
& \left.+\frac{1}{p_{1}+2} \int_{\mathbb{R}^{N}}\left|u_{n}(x)\right|^{p_{1}+2} d x\right)+\circ(1),
\end{aligned}
$$

where $a_{0}=\min \left\{2 p_{2}-2, p_{1}\right\}$. 
Note that since the series $\sum_{j=1}^{\infty}\left\|U_{\mu_{j}}^{j}\right\|_{L^{2}}^{2}$ is convergent, there exists $j_{0} \geq 1$ such that

$$
\inf _{j \geq 1} \mu_{j}=\mu_{j_{0}}=\frac{\sqrt{M}}{\left\|U^{j_{0}}\right\|_{L^{2}}} .
$$

Letting $n \rightarrow \infty$ and $l \rightarrow \infty$ in (3.11), there exists $C>0$ such that

$$
d_{M} \geq d_{M}+C\left(\left(\frac{\sqrt{M}}{\left\|U^{j_{0}}\right\|_{L^{2}}}\right)^{a_{0}}-1\right)
$$

which implies

$$
\left\|U^{j_{0}}\right\|_{L^{2}}^{2} \geq M
$$

Hence, $\left\|U^{j_{0}}\right\|_{L^{2}}^{2}=M$, and there exists only one term $U^{j_{0}} \neq 0$ in the decomposition (3.5). Moreover, we deduce from $(2.4)-(2.6)$ that $E\left(U^{j_{0}}\right)=d_{M}$. This implies that the infimum of the variational problem (3.1) is attained at $U^{j_{0}}$. This completes the proof.

Now, define

$$
S_{M}:=\left\{u \in H^{1} ; u \text { is a minimizer of the variational problem (3.1) }\right\} .
$$

Then, for any $u \in S_{M}$, we deduce from Euler-Lagrange theorem that there exists $\omega \in \mathbb{R}$ such that

$$
-\Delta u+\omega u-|u|^{p_{1}} u-\left(I_{\alpha} *|u|^{p_{2}}\right)|u|^{p_{2}-2} u=0
$$

In addition, if $u \in S_{M}$, then $u$ is a solution of (3.14), and $\psi(t, x)=e^{i \omega t} u(x)$ is a standing wave solution of (1.1). Hence $e^{i \omega t} u(x)$ is the orbit of $u(x)$. On the other hand, for any $t \geq 0$, if $u$ is a solution of (3.1), then $e^{i \omega t} u(x)$ is also solution of (3.1), that is, $e^{i \omega t} u \in S_{M}$. Applying Theorem 3.1 and the method of Cazenave and Lions [17], we will show that if the initial data is close to an orbit in the set $S_{M}$, then the solution of (1.1) remains close to the orbit in the set $S_{M}$.

Theorem 3.2 Let $N \geq 3, \lambda_{1}=-1, \lambda_{2}=-1,0<p_{1}<\frac{4}{N}, p_{2}=1+\frac{2+\alpha}{N}$, and $0<M<\|Q\|_{L^{2}}^{2}$. If Assumption 1 holds, then for arbitrary $\varepsilon>0$, there exists $\delta>0$ such that, for any $\psi_{0} \in H^{1}$ such that

$$
\inf _{u \in S_{M}}\left\|\psi_{0}-u\right\|_{H^{1}}<\delta
$$

the corresponding solution $\psi$ of (1.1) satisfies

$$
\inf _{u \in S_{M}}\|\psi(t)-u\|_{H^{1}}<\varepsilon
$$

for all $t>0$. 
Proof First, by Theorem 2.5 we see that the solution $\psi$ of (1.1) exists globally. Assume by contradiction that there exist $\varepsilon_{0}$ and a sequence $\left\{\psi_{0, n}\right\}_{n=1}^{\infty}$ such that

$$
\inf _{u \in S_{M}}\left\|\psi_{0, n}-u\right\|_{H^{1}}<\frac{1}{n}
$$

and there exists $\left\{t_{n}\right\}_{n=1}^{\infty}$ such that the corresponding solution sequence $\left\{\psi_{n}\left(t_{n}\right)\right\}_{n=1}^{\infty}$ of (1.1) satisfies

$$
\inf _{u \in S_{M}}\left\|\psi_{n}\left(t_{n}\right)-u\right\|_{H^{1}} \geq \varepsilon_{0} .
$$

From (3.15) and the conservation laws it follows that, as $n \rightarrow \infty$,

$$
\int_{\mathbb{R}^{N}}\left|\psi_{n}\left(t_{n}, x\right)\right|^{2} d x=\int_{\mathbb{R}^{N}}\left|\psi_{0, n}(x)\right|^{2} d x \rightarrow \int_{\mathbb{R}^{N}}|u(x)|^{2} d x=M
$$

and

$$
E\left(\psi_{n}\left(t_{n}\right)\right)=E\left(\psi_{0, n}\right) \rightarrow E(u)=d_{M} .
$$

Hence $\left\{\psi_{n}\left(t_{n}\right)\right\}_{n=1}^{\infty}$ is a minimizing sequence of the variational problem (3.1). We deduce from Theorem 3.1 that there exists a minimizer $\omega \in S_{M}$ such that

$$
\left\|\psi_{n}\left(t_{n}\right)-\omega\right\|_{H^{1}} \rightarrow 0, \quad n \rightarrow \infty
$$

which contradicts with (3.16). This completes the proof.

Proof of Theorem 1.1 Let $\psi_{0} \in H^{1}$ and $0<M<\|Q\|_{L^{2}}^{2}$, where $\mathrm{Q}$ is a ground state of (1.5). Then it follows from Theorem 3.1 that the variational problem (3.1) has minimizers. These minimizers correspond to the standing waves of (1.1). Therefore we obtain the existence of the standing waves of (1.1). In addition, we deduce from Theorem 3.2 and the definition of orbital stability (see [1]) that the standing waves of (1.1) are orbitally stable.

\section{Conclusions}

In this paper, we study the orbital stability of standing waves for the nonlinear Schrödinger-Choquard equation (1.1). There is no scaling invariance for this equation. When $0<$ $p_{1}<\frac{4}{N}$ and $p_{2}=1+\frac{2+\alpha}{N}$, by using variational methods and the profile decomposition of bounded sequences in $H^{1}$ we show that the standing waves are orbitally stable.

Acknowledgements

Not applicable.

Funding

This work is supported by Gansu Provincial Natural Science Foundation (18JR3RM238), the PhD scientific research start-up capital funded projects of Longdong University (XYBY05).

Abbreviations

Not applicable.

Availability of data and materials

Data sharing not applicable to this paper as no datasets were generated or analyzed during the current study. 


\section{Competing interests}

The authors declare that no competing interests exist.

\section{Authors' contributions}

The authors contributed equally to this paper. Both authors read and approved the final manuscript.

\section{Publisher's Note}

Springer Nature remains neutral with regard to jurisdictional claims in published maps and institutional affiliations.

\section{Received: 22 March 2018 Accepted: 7 October 2018 Published online: 23 October 2018}

\section{References}

1. Cazenave, T.: Semilinear Schrödinger Equations. Courant Lecture Notes in Mathematics, vol. 10. Am. Math. Soc., Providence (2003)

2. Chen, J., Guo, B.: Strong instability of standing waves for a nonlocal Schrödinger equation. Phys. D: Nonlinear Phenom. 227, 142-148 (2007)

3. d'Avenia, P., Squassina, M.: Soliton dynamics for the Schrödinger-Newton system. Math. Models Methods Appl. Sci. 24, 553-572 (2014)

4. Genev, H., Venkov, G.: Soliton and blow-up solutions to the time-dependent Schrödinger-Hartree equation. Discrete Contin. Dyn. Syst., Ser. S 5, 903-923 (2012)

5. Ginibre, J., Velo, G.: On a class of nonlinear Schrödinger equations with nonlocal interaction. Math. Z. 170, 109-136 (1980)

6. Guo, Q., Zhu, S.: Sharp threshold of blow-up and scattering for the fractional Hartree equation. J. Differ. Equ. 264, 2802-2832 (2018)

7. Liu, J., Qian, A.: Ground state solution for a Schrödinger-Poisson equation with critical growth. Nonlinear Anal., Real World Appl. 40, 428-443 (2018)

8. Mao, A., Chen, Y.: Existence and concentration of solutions for sublinear Schrödinger-Poisson equations. Indian J. Pure Appl. Math. 49, 339-348 (2018)

9. Mao, A., Yang, L., Qian, A., Luan, S.: Existence and concentration of solutions of Schrödinger-Poisson system. Appl. Math. Lett. 68, 8-12 (2017)

10. Miao, C., Xu, G., Zhao, L.: On the blow up phenomenon for the mass critical focusing Hartree equation in $\mathbb{R}^{4}$. Colloq. Math. 119, 23-50 (2010)

11. Shao, M., Mao, A.: Multiplicity of solutions to Schrödinger-Poisson system with concave-convex nonlinearities. Appl. Math. Lett. 83, 212-218 (2018)

12. Zhu, S.: On the blow-up solutions for the nonlinear fractional Schrödinger equation. J. Differ. Equ. 261, 1506-153 (2016)

13. Bonanno, C., d'Avenia, P., Ghimenti, M., Squassina, M.: Soliton dynamics for the generalized Choquard equation. J. Math. Anal. Appl. 417, 180-199 (2014)

14. Feng, B., Yuan, X.: On the Cauchy problem for the Schrödinger-Hartree equation. Evol. Equ. Control Theory 4, 431-445 (2015)

15. Feng, B.: Sharp threshold of global existence and instability of standing wave for the Schrödinger-Hartree equation with a harmonic potential. Nonlinear Anal., Real World Appl. 31, 132-145 (2016)

16. Wang, X., Sun, X., LV, W.: Orbital stability of generalized Choquard equation. Bound. Value Probl. 2016, 1 (2016)

17. Cazenave, T., Lions, P.L.: Orbital stability of standing waves for some nonlinear Schrödinger equations. Commun. Math. Phys. 85, 549-561 (1982)

18. Feng, B., Zhang, H.: Stability of standing waves for the fractional Schrödinger-Choquard equation. Comput. Math. Appl. 75, 2499-2507 (2018)

19. Feng, B., Zhang, H.: Stability of standing waves for the fractional Schrödinger-Hartree equation. J. Math. Anal. Appl. 460, 352-364 (2018)

20. Zhang, J., Zhu, S.: Stability of standing waves for the nonlinear fractional Schrödinger equation. J. Dyn. Differ. Equ. 29, $1017-1030$ (2017)

21. Zhu, S.: Existence of stable standing waves for the fractional Schrödinger equations with combined nonlinearities. J. Evol. Equ. 17, 1003-1021 (2017)

22. Feng, B.: On the blow-up solutions for the nonlinear Schrödinger equation with combined power-type nonlinearities. J. Evol. Equ. 18, 203-220 (2018)

23. Feng, B.: On the blow-up solutions for the fractional nonlinear Schrödinger equation with combined power-type nonlinearities. Commun. Pure Appl. Anal. 17, 1785-1804 (2018)

24. Lieb, E.: Analysis, 2nd edn. Graduate Studies in Mathematics, vol. 14. Am. Math. Soc., Providence (2001)

25. Moroz, V., Schaftingen, J.: Groundstates of nonlinear Choquard equations: existence, qualitative properties and decay asymptotics. J. Funct. Anal. 265, 153-184 (2013)

26. Hmidi, T., Keraani, S.: Blowup theory for the critical nonlinear Schrödinger equations revisited. Int. Math. Res. Not. 46 2815-2828 (2005) 